\title{
GENERALIZED VANDERMONDE DETERMINANTS AND ROOTS OF UNITY OF PRIME ORDER
}

\author{
R. J. EVANS AND I. M. ISAACS ${ }^{1}$
}

\begin{abstract}
Easy proofs are given for two theorems of O. $\mathrm{H}$. Mitchell about a type of generalized Vandermonde determinant. One of these results is then used to prove that if $|F(\varepsilon): F|=n$ where $F$ is a field of characteristic zero and $\varepsilon$ is a root of unity of prime order, then every set of $n$ powers of $\varepsilon$ forms an $F$-basis for $F(\varepsilon)$.
\end{abstract}

1. Introduction and notation. Let $\mathbf{a}=\left(a_{1}, \ldots, a_{n}\right)$ be an $n$-tuple of distinct nonnegative integers and let $V_{\mathbf{a}}\left(X_{1}, \ldots, X_{n}\right)$ be the polynomial obtained by computing the determinant of the matrix with $(i, j)$ entry equal to $X_{i}^{a_{j}}$ where the $X_{i}$ are indeterminates. We fix the "standard" $n$-tuple $s=(0,1, \ldots, n-1)$ so that

$$
V_{s}\left(X_{1}, \ldots, X_{n}\right)=\prod_{1 \leqslant i<j \leqslant n}\left(X_{j}-X_{i}\right)
$$

is the Vandermonde determinant.

Note that $V_{\mathrm{a}}$ is divisible by $V_{\mathbf{s}}$ in the polynomial ring $\mathbf{Z}\left[X_{1}, \ldots, X_{n}\right]$. We write $P_{\mathrm{a}}=V_{\mathrm{a}} / V_{\mathbf{s}}$. Observe that $P_{\mathrm{a}}$ is a homogeneous polynomial since both $V_{\mathrm{a}}$ and $V_{\mathbf{s}}$ are.

Under the hypothesis that $0 \leqslant a_{1}<a_{2}<\cdots<a_{n}$, O. H. Mitchell [1] proved the striking result that all of the coefficients of $P_{\mathrm{a}}$ are nonnegative. (Contrast this with the fact that for $n>1$, only half of the nonzero coefficients of $V_{\mathrm{a}}$ are positive.)

Under the same hypothesis, Mitchell proved that $P_{\mathrm{a}}$ has exactly $V_{s}\left(a_{1}, a_{2}, \ldots, a_{n}\right) / V_{s}(0,1, \ldots, n-1)$ "terms", i.e. the sum of the coefficients of $P_{\mathbf{a}}$ is $V_{\mathbf{s}}(\mathbf{a}) / V_{\mathbf{s}}(\mathbf{s})$.

In $\$ 2$ of this paper we give simple new proofs of Mitchell's theorems and in $\$ 3$ we use information about $V_{\mathrm{a}}$ to prove the following result.

THEOREM 1. Let $F$ be a field of characteristic zero and let $|F(\varepsilon): F|=n$ where $\varepsilon$ is a pth root of unity for some prime $p$. Then every set of $n$ distinct powers of $\varepsilon$ is a basis for $F(\varepsilon)$ over $F$.

\section{Mitchell's theorems.}

Lemma 2. Let $\mathbf{a}=\left(a_{1}, \ldots, a_{n}\right)$ with $0=a_{1}<a_{2}<\cdots<a_{n}$ and let $\mathscr{B}(\mathbf{a})$

Received by the editors September 2, 1975.

AMS (MOS) subject classifications (1970). Primary 12A35, 15A15.

Key words and phrases. Vandermonde determinant, roots of unity.

1 The second author's work was partially supported by NSF Grant GP42457X. 
denote the following set of $(n-1)$-tuples: $\left\{\left(b_{1}, \ldots, b_{n-1}\right) \mid a_{i} \leqslant b_{i}<a_{i+1}\right\}$. Then

$$
V_{\mathbf{a}}\left(1, X_{2}, \ldots, X_{n}\right)=\prod_{i=2}^{n}\left(X_{i}-1\right) \sum_{\mathbf{b} \in \mathscr{B}(\mathbf{a})} V_{\mathbf{b}}\left(X_{2}, \ldots, X_{n}\right) .
$$

Proof. Since $a_{1}=0$, we have that $V_{\mathrm{a}}\left(1, X_{2}, \ldots, X_{n}\right)$ is the determinant of a matrix with all entries in the first row and column equal to 1 . Subtract the first row from each of the others and expand by minors on the first column. It follows that

$$
V_{\mathrm{a}}\left(1, X_{2}, \ldots, X_{n}\right)=\operatorname{det}\left(X_{i}^{a_{j}}-1\right)
$$

where the row and column indices $i$ and $j$ run from 2 to $n$. Next, factor $X_{i}-1$ from the $i$ th row. This yields

$$
V_{\mathrm{a}}\left(1, X_{2}, \ldots, X_{n}\right)=\prod_{i=2}^{n}\left(X_{i}-1\right) \cdot \Delta
$$

where $\Delta$ is the $(n-1) \times(n-1)$ determinant with $(i, j)$ entry equal to $\sum_{\nu=0}^{a_{j}-1} X_{i}^{\nu}$ for $2 \leqslant i, j \leqslant n$.

To compute $\Delta$, subtract column $j-1$ from column $j$ for $j=n, n-1, \ldots$, 3. The $(i, j)$ entry of what results is $\sum_{\nu=a_{j-1}}^{a_{j}-1} X_{i}^{\nu}$ where $i$ and $j$ still run between 2 and $n$. Expansion by linearity on the columns now yields

$$
\Delta=\sum_{\mathbf{b} \in \mathscr{B}(\mathbf{a})} V_{\mathbf{b}}\left(X_{2}, \ldots, X_{n}\right)
$$

and the proof is complete.

Lemma 3. Let $\mathbf{a}=\left(a_{1}, a_{2}, \ldots, a_{n}\right)$ with $0=a_{1}<a_{2}<\cdots<a_{n}$ and let $\mathscr{B}(\mathbf{a})$ be as in Lemma 2. Then

$$
P_{\mathbf{a}}\left(1, X_{2}, \ldots, X_{n}\right)=\sum_{\mathbf{b} \in \mathscr{B}(\mathbf{a})} P_{\mathbf{b}}\left(X_{2}, \ldots, X_{n}\right)
$$

Proof. Since $\mathscr{B}(\mathbf{s})$ contains only the standard $(n-1)$-tuple $(0,1, \ldots, n$ - 2), the result follows upon application of Lemma 2 to both the numerator and denominator of $V_{\mathrm{a}} / V_{\mathrm{s}}=P_{\mathrm{a}}$.

TheOREM 4 (Mitchell). Let $\mathbf{a}=\left(a_{1}, \ldots, a_{n}\right)$ with $0 \leqslant a_{1}<\cdots<a_{n}$. Then all of the coefficients of $P_{\mathrm{a}}$ are nonnegative.

Proof. We may factor out $\left(X_{1} X_{2} \cdots X_{n}\right)^{a_{1}}$ from $P_{\mathrm{a}}$ and it is thus no loss to assume that $a_{1}=0$. If $\mathbf{b}=\left(b_{1}, b_{2}, \ldots, b_{n-1}\right) \in \mathscr{B}(\mathbf{a})$, then $0 \leqslant b_{1}<b_{2}$ $<\cdots<b_{n-1}$ and so all coefficients of $P_{\mathbf{b}}\left(X_{2}, \ldots, X_{n}\right)$ are nonnegative, by induction on $n$. By Lemma 3 , therefore, the coefficients of $P_{a}\left(1, X_{2}, \ldots, X_{n}\right)$ (viewed as a polynomial in $n-1$ indeterminates) are all nonnegative. Since $P_{a}\left(X_{1}, \ldots, X_{n}\right)$ is homogeneous, the result follows.

Theorem 5 (Mitchell). Let $\mathbf{a}=\left(a_{1}, \ldots, a_{n}\right)$. Then the sum of the coefficients of $P_{\mathbf{a}}$ equals $V_{\mathbf{s}}\left(a_{1}, \ldots, a_{n}\right) / V_{\mathbf{s}}(0,1, \ldots, n-1)$.

Proof. We have 


$$
\begin{aligned}
& P_{\mathbf{a}}\left(1, X, X^{2}, \ldots, X^{n-1}\right) V_{\mathbf{s}}\left(1, X, X^{2}, \ldots, X^{n-1}\right) \\
& \quad=V_{\mathbf{a}}\left(1, X, X^{2}, \ldots, X^{n-1}\right)=V_{\mathbf{s}}\left(X^{a_{1}}, X^{a_{2}}, \ldots, X^{a_{n}}\right)
\end{aligned}
$$

where the last equality follows since the two polynomials are determinants of transposed matrices.

Now if $u$ and $v$ are distinct nonnegative integers, then the polynomial $\left(X^{u}-X^{v}\right) /(X-1)$ takes on the value $u-v$ when $X=1$. Since

$$
V_{\mathbf{s}}\left(X^{b_{1}}, \ldots, X^{b_{n}}\right)=\prod_{i<j}\left(X^{b_{j}}-X^{b_{i}}\right)
$$

it follows for any $n$-tuple of distinct nonnegative integers $\left(b_{1}, b_{2}, \ldots, b_{n}\right)$, that the polynomial

$$
V_{\mathbf{s}}\left(X^{b_{1}}, \ldots, X^{b_{n}}\right) /(X-1)^{\left(\begin{array}{c}
n \\
2
\end{array}\right)}
$$

takes on the value $V_{\mathbf{s}}\left(b_{1}, \ldots, b_{n}\right)$ when $X=1$. Thus dividing both sides of (1) by

$$
(X-1)^{\left(\frac{n}{2}\right)}
$$

and setting $X=1$, we obtain

$$
P_{\mathbf{a}}(1, \ldots, 1)=V_{\mathbf{s}}\left(a_{1}, \ldots, a_{n}\right) / V_{\mathbf{s}}(0,1, \ldots, n-1)
$$

and the result follows.

\section{Powers of $\varepsilon$.}

THEOREM 6. Let $p$ be a prime and let $\varepsilon$ be a pth root of unity in some field of characteristic zero. Suppose $a_{1}, \ldots, a_{n} \in \mathbf{Z}$ are pairwise incongruent $(\bmod p)$ and suppose the same for $b_{1}, \ldots, b_{n} \in \mathbf{Z}$. Then $\operatorname{det}\left(\varepsilon^{a_{i} b_{j}}\right) \neq 0$.

Proof. We may assume without loss that all $a_{i} \geqslant 0$ and we let a $=\left(a_{1}, \ldots, a_{n}\right)$. We need to show that $V_{\mathbf{a}}\left(\varepsilon^{b_{1}}, \ldots, \varepsilon^{b_{n}}\right) \neq 0$. Since the $\varepsilon^{b_{i}}$ are distinct, we have that $V_{\mathbf{s}}\left(\varepsilon^{b_{1}}, \ldots, \varepsilon^{b_{n}}\right) \neq 0$ and thus it suffices to show that $P_{\mathbf{a}}\left(\varepsilon^{b_{1}}, \ldots, \varepsilon^{b_{n}}\right) \neq 0$.

Now if $f \in \mathbf{Z}[X]$ with $f(\varepsilon)=0$, then $f$ is divisible by $X^{p-1}+\cdots+X+1$ in $\mathbf{Z}[X]$ and thus $f(1) \equiv 0(\bmod p)$. If $P_{\mathbf{a}}\left(\varepsilon^{b_{1}}, \ldots, \varepsilon^{b_{n}}\right)=0$, it follows that $P_{\mathbf{a}}(1,1, \ldots, 1) \equiv 0(\bmod p)$ and thus by Theorem $5,0 \equiv V_{\mathbf{s}}\left(a_{1}, \ldots, a_{n}\right)$ $=\prod_{i<j}\left(a_{j}-a_{i}\right)(\bmod p)$. This contradicts the hypothesis on the $a_{i}$ and completes the proof.

We now prove the theorem stated in $\S 1$.

Proof of TheOrem 1. We may assume that $n>1$ and thus $\varepsilon$ is a primitive $p$ th root of unity. Let $\varepsilon^{a_{1}}, \ldots, \varepsilon^{a_{n}}$ be distinct and assume that

$$
\sum \alpha_{i} \varepsilon^{a_{i}}=0 \quad\left(\alpha_{i} \in F\right) .
$$

Let $G=\operatorname{Gal}(F(\varepsilon) / F)$. Then $\varepsilon$ has $n$ distinct images $\varepsilon^{b_{1}}, \varepsilon^{b_{2}}, \ldots, \varepsilon^{b_{n}}$ under $G$. Application of the elements of $G$ to (2) yields that $\sum_{i=1}^{n} \alpha_{i} \varepsilon^{a_{i} b_{j}}=0$ for all $j$. Since $\operatorname{det}\left(\varepsilon^{a_{i} b_{j}}\right) \neq 0$ by Theorem 6 , we conclude that all $\alpha_{i}=0$. The result now follows. 
COROllary 7. Let $F$ have characteristic zero and let $p$ be a prime. Suppose $f \in F[X]$ is irreducible of degree $n$ and divides $X^{p}-1$. Then all $n+1$ coefficients of $f$ are nonzero.

Proof. Otherwise, $f$ provides a nontrivial dependence relation on $n$ powers of $\varepsilon$, contradicting Theorem 1 .

We close with the remark that Corollary 7 does not remain true if the hypothesis that $F$ has characteristic zero is dropped. For instance, over $G F(2)$, the polynomial $X^{3}+X+1$ is irreducible and divides $X^{7}-1$. It follows that Theorem 6 and Theorem 1 are also false for general fields.

\section{REFERENCES}

1. O. H. Mitchell, Note on determinants of powers, Amer. J. Math. 4 (1881), 341-344.

Department of Mathematics, University of California at San Diego, La Jolla, CaliforNIA 92037

Department of Mathematics, University of Wisconsin, Madison, Wisconsin 53706 